\title{
Vitamina C como antioxidante en el manejo del SARS-CoV-2
}

\section{Vitamin $C$ as antioxidant in SARS-CoV-2 management}

\author{
Mejía $L^{1}$, Alvarado $A^{1}$
}

${ }^{1}$ Endocrinóloga, EPS Sánitas. Miembro de número, Asociación Colombiana de Endocrinología, Bogotá, Colombia.

Autor de correspondencia: Liliana Mejía Vidal

Correo electrónico: liliana@doctoramejia.com

Autor de correspondencia: Alejandra Alvarado Ospina

Correo electrónico: alejitaalvarado@hotmail.com

\section{Resumen}

La situación actual de la pandemia generada por el nuevo coronavirus SARS-CoV-2, por su morbimortalidad, ha hecho que la comunidad científica esté implementando diferentes estrategias de tratamientos para su manejo. Es el caso de la vitamina $\mathrm{C}$, principal antioxidante no enzimático en el plasma (1) , la cual se ha usado para la prevención de infecciones respiratorias y para el tratamiento de la sepsis en pacientes críticos. El presente artículo pretende revisar la evidencia en torno al papel de la vitamina $\mathrm{C}$ como posible coadyuvante en el manejo de pacientes con infección por SARS-CoV-2.

Palabras clave: COVID-19, vitamina C, antioxidante.

\section{Abstract}

The current situation of pandemic generated by the new coronavirus SARS-CoV-2, by its morbid-mortality, has caused the scientific community to be implementing different treatment strategies for its management. This is the case of vitamin $\mathrm{C}$, the main non-enzymatic antioxidant in plasma ${ }^{(1)}$, which has been used for the prevention of respiratory infections and for the treatment of sepsis in critical patients. This article aims to review the evidence surrounding the role of vitamin $\mathrm{C}$ as a possible adjuvant in the management of patients with SARS-CoV-2 infection.

Keywords: COVID 19, Vitamin C, Antioxidant.

\section{Introducción}

En la sepsis se generan especies reactivas de oxígeno y de nitrógeno, que conducen a la activación de los factores de transcripción que inducen la oxidación, como las citocinas y las quimiocinas proinflamatorias ${ }^{(2)}$. Esto causa daño endotelial, permeabilidad vascular, activación de neutrófilos y adhesión plaquetaria en la microvasculatura ${ }^{(3)}$. En pacientes críticos se ha documentado la depleción de los niveles de séricos de vitamina $\mathrm{C}$ y existe una correlación inversa con la falla multiorgánica ${ }^{(4)}$ y directa con las tasas de supervivencia ${ }^{(5)}$.

Son claros los múltiples mecanismos de lesión tisular por sepsis, aunque no hay un tratamiento más allá del manejo antibiótico y de sostén. Existe evidencia reciente del uso de la vitamina $\mathrm{C}$ como un tratamiento adicional en sepsis y en infección respiratoria. Por lo anterior, dada la infección por COVID-19, se ha planteado el uso de vitamina C.

\section{Infección por COVID-19 en el pulmón}

En la infección por COVID-19 se ha evidenciado un daño alveolar difuso, asociado a una hiperplasia de células epiteliales pulmonares y macrófagos activados alveolares e intersticiales. También se ha documentado un retraso de la respuesta del interferón (IFN) tipo 1, que genera un aumento de los macrófagos inflamatorios patogénicos y de la apoptosis de células T. Estos macrófagos activados y las células $\mathrm{T}$ efectoras tienen una tasa de glucólisis alta, que es llamada efecto Warburg. Esto produce disociación de la enzima gliceraldehído-3-fosfato deshidrogenasa (GAPDH) con el ARNm del interferón gamma (IFN- $\gamma$ ), que induce activación de las células T y que, en situaciones normales, se une al ARNm del IFN- $\gamma$, inhibiendo su traducción. Adicionalmente, Los macrófagos activados producen grandes cantidades de lactato, el cual es ingresado a las células epiteliales alveolares tipo II por el transportador MCT1 
y esto, a su vez, atenúa la respuesta inmunitaria innata por la disminución de la producción de IFN tipo I para el aclaramiento viral ${ }^{(6)}$

\section{Vitamina C: farmacología}

La vitamina $\mathrm{C}$ o L-ácido ascórbico es una vitamina hidrosoluble, cofactor esencial de diversas reacciones enzimáticas que median funciones biológicas esenciales. También es considerada un potente antioxidante, con propiedades antiinflamatoria y antimicrobiana. Es encontrada en frutas cítricas, pimentón verde, brócoli, fresa y papas, no es sintetizada por el humano y debe ser adquirida a través de la dieta ${ }^{(7)}$.Se absorbe en las células del epitelio del intestino delgado, a través del transportador de vitamina C dependiente de sodio (SVCT1). También en la membrana apical del epitelio intestinal, una forma oxidada de la vitamina C, el ácido dehidroascórbico (DHA), es transportada hacia el interior de la célula por el glucotransportador 2 (GLUT2) y el GLUT8. Luego de la absorción intestinal, la vitamina $\mathrm{C}$ se distribuye en la sangre y en los compartimentos extracelulares, y, a través del transportador SVCT2, es utilizada por los diferentes tejidos del cuerpo. Finalmente, la vitamina $\mathrm{C}$ es filtrada libremente por el riñón y reabsorbida por el SVCT1 en el túbulo contorneada proximal. La biodisponibilidad de la vitamina C, por vía oral, alcanza el 100\% con una única dosis de $200 \mathrm{mg}$, aunque solo alcanza el 33\% con una dosis oral de $1250 \mathrm{mg}$. Esto sucede porque en el intestino, la proteína del SVCT1 es saturable. Para saltar el paso intestinal del SVCT1, se ha administrado vitamina C intravenosa (IV), lográndose concentraciones 70 veces más elevadas en plasma, comparadas con las alcanzadas con la administración oral ${ }^{(2)}$.

\section{Vitamina C en la infección por COVID-19}

En la actualidad, la vitamina C desempeña un papel importante en el manejo de la infección generada por la COVID-19, en donde se presenta una activación macrofagocítica, que genera una tormenta de citocinas proinflamatorias que reacciona con las células endoteliales del pulmón y produce una infiltración de neutrófilos, lo que aumenta el estrés oxidativo y lleva a daño en la función de la barrera pulmonar. Las altas dosis de vitamina $\mathrm{C}$ inhiben la GAPDH, disminuyendo la generación de ATP y piruvato, lo que induce una crisis energética que, finalmente, conduce a la muerte celular ${ }^{(5,8)}$.

Las células epiteliales pulmonares utilizan la fosforilación oxidativa mitocondrial para producir ATP; por lo tanto, el tratamiento con altas dosis de vitamina $\mathrm{C}$ actúa como un antioxidante para las células epiteliales pulmonares ${ }^{(6)}$.

El tratamiento con vitamina $\mathrm{C}$ puede proteger la inmunidad innata de las células epiteliales alveolares tipo II, mediante la inhibición de la secreción de lactato producida por las células inmunitarias activadas ${ }^{(6)}$. También actúa como cofactor de las enzimas alfa-cetoglutarato dioxigenasas, que son enzimas que participan en la síntesis de neurotransmisores en la regulación de la expresión génica y en la reticulación de las fibras de colágeno. ${ }^{(5,7)}$.

\section{Evidencia}

El ensayo de CRITIS-ALI fue un ensayo multicéntrico, doble ciego, controlado, que inscribió a 167 pacientes con sepsis y síndrome de dificultad respiratoria aguda (SDRA), que fueron aleatorizados para recibir $50 \mathrm{mg} / \mathrm{kg}$ cada 6 horas, en dosis altas de vitamina C IV durante 4 días versus placebo. Este estudio mostró una diferencia significativa en la mortalidad por todas las causas a los 28 días $(29,8 \%$ en el grupo de infusión de vitamina C IV versus $46,3 \%$ en el grupo placebo, con una $p<0,05)$; además, el grupo tratado con vitamina $\mathrm{C}$ mostró más días libres de la unidad de cuidados intensivos (UCI) en el día $28(p=0,03)$, menos días de hospitalización $(22,6 \%$ con vitamina C IV versus $15,5 \%$ con placebo [ $p=0,04]$ ) y menos días de ventilación mecánica (13,1\% con vitamina C IV versus $10,6 \%$ con placebo $[p=0,15])$. Dado los resultados publicados en este estudio, dentro de los protocolos de tratamiento instaurados en el manejo del paciente con infección por COVID-19 se incluye el manejo con vitamina $C^{(5)}$. Este estudio revela que los altos niveles plasmáticos de vitamina $\mathrm{C}$ actúan de forma "pleiotrópica" para atenuar la inflamación sistémica y corregir las anomalías de la coagulación inducidas por la sepsis, al tiempo que atenúan la lesión vascular (ensayo clínico CITRIS-ALI; identificador: NCT02106975).

Estudios previos, como el de Nathens y colaboradores, en donde administraron $1 \mathrm{~g}$ de ácido ascórbico cada 8 horas combinado con vitamina $\mathrm{E}$ oral, durante 28 días, en 594 pacientes quirúrgicamente críticos, mostró una incidencia significativamente menor de lesión pulmonar aguda y falla multiorgánica ${ }^{(9)}$. Otros estudios realizados con animales han demostrado que la vitamina $\mathrm{C}$ aumenta la resistencia a la infección causada por el coronavirus y también modifica la susceptibilidad a la infección ${ }^{(10)}$.

En China, dosis altas intravenosas han sido administradas con éxito en el tratamiento de 50 pacientes infectados por COVID-19; las dosis utilizadas variaron entre 10 g y 20 g por día, administrados durante un período de 8 a 10 horas ${ }^{(11,12)}$.

Dada esta experiencia, el uso temprano de grandes dosis de antioxidantes como la vitamina $\mathrm{C}$ se ha incluido en el tratamiento de la COVID-19 y se está utilizando como medida preventiva en poblaciones susceptibles, como los trabajadores de la salud con mayores riesgos de exposición.

\section{Protocolo de administración}

Según la evidencia clínica, se han utilizado diferentes protocolos de administración endovenosa de la vitamina C. Los estudios muestran un mayor beneficio en los primeros períodos de la enfermedad. El régimen propuesto inicia con la 
administración de $50 \mathrm{mg} / \mathrm{kg}$ de peso corporal, cada 6 horas, durante 4 días, con una restricción de glucosa y una velocidad de infusión de 0,25-0,5 g/min. En algunos protocolos se menciona la utilidad de los glucocorticoides, dada la preocupación de que las dosis altas de vitamina $\mathrm{C}$ produzcan una muerte celular osmótica de las células inmunitarias, pero no la apoptosis, que podría generar una inflamación local en el medio alveolar, de tal manera que, al agregar glucocorticoides IV, se podrá atenuar las posibles complicaciones inflamatorias del tratamiento, por lo que se sugiere la aplicación de hidrocortisona en 50 mg IV, cada 6 horas, durante 7 días. Así mismo, se recomienda tener en cuenta el efecto quelante de la vitamina $\mathrm{C}$, lo que puede llevar a hipocalcemia e hipomagnesemia, por lo que es un dato que debe considerarse para la medición de estos electrólitos durante el tratamiento ${ }^{(6,7)}$.

\section{Conclusiones}

La infección por coronavirus genera un síndrome de dificultad respiratoria aguda de rápida progresión, que aumenta la mortalidad de una manera importante en los pacientes infectados, sin tener, a la fecha, una vacuna contra este virus. Por tanto, se han creado protocolos de manejo con múltiples tera- pias, entre estos, un potente antioxidante de bajo costo, como lo es la vitamina $C$, que ha mostrado efectividad en pacientes con sepsis grave, y pese a que cuenta con pocos reportes realizados hasta el momento, ha sido efectiva en pacientes con infección por COVID-19, siendo una alternativa segura.

El efecto prooxidante de la vitamina $\mathrm{C}$ se ha considerado para el manejo de los pacientes con infección por COVID-19, dada su potente actividad antiinflamatoria, que disminuye la cascada de citocinas activas a nivel del endotelio capilar alveolar.

Faltan estudios a futuro donde se demuestre el efecto in vivo del uso de la vitamina $\mathrm{C}$ en los pacientes con infección por COVID-19 y, de esta manera, precisar las dosis adecuadas para el tratamiento de estos pacientes.

\section{Conflictos de interés \\ Ninguno.}

\section{Financiación}

Ninguna.

\section{Agradecimientos}

Agradecemos a la Asociación Colombiana de Endocrinología por darnos la oportunidad de publicar sobre este tema.

\section{Referencias}

1. Carr AC, Maggini S. Vitamin $\mathrm{C}$ and Immune Function. Nutrients. 2017;9(11):1211. doi: $10.3390 /$ nu9111211.

2. Kashiouris MG, L'Heureux M, Cable CA, Fisher BJ, Leichtle SW, Fowler AA. The Emerging Role of Vitamin $\mathrm{C}$ as a Treatment for Sepsis. Nutrients. 2020;12(2):292. doi: 10.3390/nu12020292.

3. Marshall JC. Clinical trials of mediator-directed therapy in sepsis: What have we learned? Intensive Care Med. 2000;26(Suppl. 1):S75-S83. doi: $10.1007 / \mathrm{s} 001340051122$

4. Borrelli E, Roux-Lombard P, Grau GE, Girardin E, Ricou B, Dayer J, et al. Plasma concentrations of cytokines, their soluble receptors, and antioxidant vitamins can predict the development of multiple organ failure in patients at risk. Crit Care Med. 1996;24(3):392-7. doi: 10.1097/00003246$199603000-00006$.

5. Fowler AA, Truwit JD, Hite RD, Morris PE, DeWilde C, Priday A, et al. Effect of Injury in Patients With Sepsis and Severe Acute Respiratory Failure: The CITRIS-ALI Randomized Clinical Trial. JAMA. 2019;322(13):1261-70. doi: 10.1001/jama.2019.11825.

6. Erol A. High-dose intravenous vitamin C treatment for COVID-19 [Internet]. Disponible en: https://bit.ly/3coGczw.
7. Hernández A, Papadakos PJ, Torres A, González DA, Vives M, Ferrando C, et al. Dos terapias conocidas podrían ser efectivas como adyuvantes en el paciente crítico infectado por COVID-19. Rev Esp Anestesiol Reanim. 2020;67(5):245-52. doi: https://doi.org/10.1016/j.redar.2020.03.004

Yun J, Mullarky E, Lu C, Bosch KN, Kavalier A, Rivera K, et al. Vitamin C selectively kills KRAS and BRAF mutant colorectal cancer cells by targeting GAPDH. Science. 2015;350(6266):1391-6. doi: 10.1126/science.aaa5004.

8. Nathens AB, Neff MJ, Jurkovich GJ, Klotz P, Farver K, Ruzinski JT, et al. Randomized, prospective trial of antioxidant supplementation in critically III surgical patients. Ann Surg. 2002;236(6):814-22. doi: 10.1097/00000658200212000-00014.

9. Zhujiang Hospital. Hydrocortisone, Vitamin C, and Thiamine for the Treatment of Sepsis and Septic Shock (HYVCTTSSS) [Internet]. Disponible en: https:// clinicaltrials.gov/ct2/show/NCT03258684.

10. National Cancer Institute. High-dose vitamin $C\left(\mathrm{PDQ}^{\mathbb{}}\right)$ - Health Professional Version [Internet]. Disponible en: https://bit.ly/3dmOCsm.

11. Zhang M, Jativa DF. Vitamin C supplementation in the critically ill: A systematic review and meta-analysis. SAGE Open Med. 2018;6:2050312118807615. doi: $10.1177 / 2050312118807615$. 